\title{
Öffentliche Gesundheit im sozialen Spannungsfeld
}

\author{
Public Health in Social Network
}

\author{
Autor \\ B. Jaeschke \\ Institut \\ Gesundheits- und Umweltamt Hamburg
}

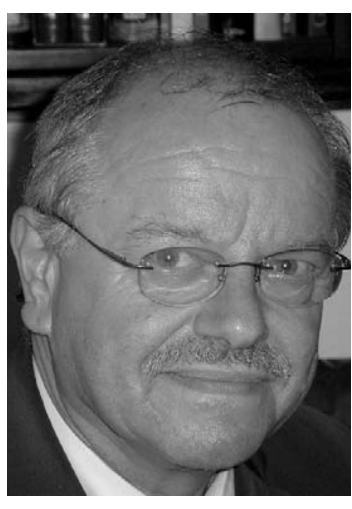

Dr. Burkhardt Jaeschke
Liebe Leserinnen und Leser,

Jahr für Jahr richtet der Berufsverband der Ärzte und Zahnärzte des ÖGD (wobei im Arztbegriff selbstverständlich die weibliche Form inkludiert ist) eine zentrale Fortbildungsveranstaltung aus - den Wissenschaftlichen Kongress. In diesem Jahr fand der 58. Ende Mai im niedersächsischen Braunschweig statt, er stand unter dem Motto: „Öffentliche Gesundheit im sozialen Spannungsfeld“".

Ein Motto, das aus meiner Sicht hervorragend in die zeitgeschichtlichen Entwicklungen, Veränderungen, ja Verwerfungen unserer sozialen und gesundheitlichen Sicherungssysteme passt, die auch zunehmend kürzeren Halbwertszeiten zu unterliegen scheinen, wenn man bedenkt, dass allein in den vergangenen vierzehn Jahren bereits fünf sogenannte Gesundheitsreformen über uns gekommen sind.

Die Kongresse bieten die Gelegenheit, neue Entwicklungen im breiten Spektrum des Öffentlichen Gesundheitsdienstes darzustellen, der ebenfalls in den letzten 14 Jahren eine erhebliche Veränderung seiner Aufgaben erfahren hat. Neben den klassischen Aufgaben des amtsärztlichen Gutachtenwesens haben neue Aufgaben in der Gesundheitsberichterstattung, bei der Prävention im Kinder- und Jugendärztlichen Dienst, beim Infektionsschutz, in der Umweltmedizin, in der sozialpsychiatrischen Versorgung und bei der primärpräventiven zahnmedizinischen Betreuung immer größere Bedeutung gewonnen.

Fragen der Methodik und des Qualitätsmanagements, der komplementären Versorgung und nicht zuletzt der Vernetzung mit anderen Akteuren unseres Sozial- und Gesundheitswesens stehen heute im Vordergrund.

Das große Interesse an diesen Themen kann aus der hohen Teilnehmerzahl (über 700!) abgelesen werden, rund 90 Vortragsveranstaltungen und Workshops sowie über 50 wissenschaftliche Poster haben die Mitarbeiterinnen und Mitarbeiter aus dem öffentlichen Gesundheitsdienst nach Braunschweig gelockt.

Es ist bereits gute Tradition, dass in dieser Zeitschrift neben den eingereichten Abstracts, die zum Kongressbeginn allen Teilnehmern ausgehändigt werden, eine Auswahl von Publikationen der Autoren aus den genannten Fachrichtungen des ÖGD abgedruckt werden, die auf dem jeweiligen Kongress vorgetragen haben. Es soll den Leserinnen und Lesern, die nicht teilnehmen konnten, ermöglichen, zumindest einen Eindruck von der Vielfalt der wissenschaftlichen Beiträge zu erhalten, für diejenigen, die teilgenommen haben, mag dieses Heft zur Nachbereitung und Reminiszenz dienen.

Allen Autoren, insbesondere auch denjenigen, die nicht berücksichtigt werden konnten, sei an dieser Stelle sehr herzlich für die zusätzliche Mühe gedankt, die sie sich auferlegt haben.
Gesundheitswesen 2008 ;

70: 623

(c) Georg Thieme Verlag KG

Stuttgart · New York

ISSN 0941-3790

Korrespondenzadresse

\section{Dr. B. Jaeschke}

Behörde für Soziales, Familie, Gesundheit und Verbraucherschutz Hamburg

Am Irrgarten 7

21073 Hamburg

burkhardt.jaeschke@bsg.

hamburg.de 